\title{
SLICES IN THE UNIT BALL OF A UNIFORM ALGEBRA
}

\author{
OLAV NYGAARD AND DIRK WERNER
}

\begin{abstract}
We show that every nonvoid relatively weakly open subset, in particular every slice, of the unit ball of an infinite-dimensional uniform algebra has diameter 2 .
\end{abstract}

It is an important task in Banach space theory to determine the extreme point structure of the unit ball for various examples of Banach spaces. The most common way to describe "corners" of convex sets is by looking for extreme points, exposed points, denting points and strongly exposed points. Every strongly exposed point is both denting and exposed and every denting or exposed point is extreme.

In this note $\mathcal{A}$ denotes an infinite-dimensional uniform algebra, i.e., an infinite-dimensional closed subalgebra of some $C(K)$-space which separates the points of $K$ and contains the constant functions. In 11 Beneker and Wiegerinck demonstrated the non-existence of strongly exposed points in $B_{\mathcal{A}}$, the closed unit ball of $\mathcal{A}$. Here we shall prove a stronger result by more elementary means. A corollary of our result is that the set of denting points is, in fact, also empty.

Recall that we may assume that $K$ is the Silov boundary of $\mathcal{A}$. It is a fundamental result in the theory of uniform algebras that then the set of strong boundary points is dense in $K$; cf. [4, p. 48 and p. 78]. (A point $x \in K$ is a strong boundary point if for every neighbourhood $V$ of $x$ and every $\delta>0$ there is some $f \in \mathcal{A}$ such that $f(x)=\|f\|=1$ and $|f| \leq \delta$ off V.)

We now turn to our first result, which gives a quantitative statement of the non-dentability of $B_{\mathcal{A}}$.

Theorem 1. Every slice of the unit ball of an infinite-dimensional uniform algebra $\mathcal{A}$ has diameter 2 .

Proof. Take an arbitrary slice $S=\left\{a \in B_{\mathcal{A}}\right.$ : re $\left.\ell(a) \geq 1-\varepsilon\right\}$, where $\|\ell\|=1$. We will produce two functions in $S$ having distance nearly 2 .

Let $0<\delta \leq \varepsilon / 11$. We first pick some $f \in B_{\mathcal{A}}$ such that

$$
\operatorname{re} \ell(f) \geq 1-\delta \text {. }
$$

The functional $\ell$ can be represented by a regular Borel measure $\mu$ on $K$ with $\|\mu\|=1$, i.e., $\ell(a)=\int_{K} a d \mu$ for all $a \in \mathcal{A}$. Let $\emptyset \neq V_{0} \subset K$ be an open set with $|\mu|\left(V_{0}\right) \leq \delta$; such a set exists since $K$ is infinite. Fix a strong boundary point $x_{0} \in V_{0}$. Using the definition of a strong boundary point, inductively construct functions $g_{1}, g_{2}, \ldots \in \mathcal{A}$ and nonvoid open subsets

Date: November 6, 2017.

1991 Mathematics Subject Classification. Primary: 46B20.

Key words and phrases. Uniform algebra, slice, denting point. 
$V_{0} \supset V_{1} \supset V_{2} \supset \ldots$ such that

$$
g_{n}\left(x_{0}\right)=\left\|g_{n}\right\|=1, \quad\left|g_{n}\right| \leq \delta \text { on } K \backslash V_{n-1}
$$

and

$$
V_{n}=\left\{x \in V_{n-1}:\left|g_{n}(x)-1\right|<\delta\right\} .
$$

Let $N>1 / \delta$ and define

$$
g=\frac{1}{N} \sum_{k=1}^{N} g_{k}, \quad h=f(1-g) \in \mathcal{A}
$$

By construction, $|h| \leq \delta$ on $V_{N}$ and $|h| \leq 1+\delta$ on $K \backslash V_{0}$. We claim that $\|h\| \leq 1+3 \delta$. In fact, if $x \in V_{r-1} \backslash V_{r}$, then $\left|1-g_{k}(x)\right| \leq \delta$ if $1 \leq k<r$, $\left|g_{r}(x)\right| \leq 1$ and $\left|g_{k}(x)\right| \leq \delta$ if $r<k \leq N$, and therefore

$$
|h(x)| \leq \frac{1}{N} \sum_{k=1}^{N}\left|1-g_{k}(x)\right| \leq \frac{(N-1)(1+\delta)+2}{N} \leq 1+3 \delta .
$$

We now estimate $|\ell(f)-\ell(h)|$ :

$$
\begin{aligned}
|\ell(f)-\ell(h)| & \leq \int_{K \backslash V_{0}}|f-h| d|\mu|+\int_{V_{0}}|f-h| d|\mu| \\
& \leq \int_{K \backslash V_{0}}|g| d|\mu|+\int_{V_{0}}(|f|+|h|) d|\mu| \\
& \leq \delta+(2+3 \delta)|\mu|\left(V_{0}\right) \leq 4 \delta .
\end{aligned}
$$

Next, we produce a function $\varphi \in \mathcal{A}$ such that

$$
\varphi\left(x_{0}\right)=\|\varphi\|=1, \quad|\varphi| \leq \delta \text { on } K \backslash V_{N} .
$$

We then have $\|h \pm \varphi\| \leq 1+4 \delta$, and the functions $\psi_{ \pm}=(h \pm \varphi) /(1+4 \delta)$ are in the unit ball of $\mathcal{A}$. We have $|\ell(\varphi)| \leq 2 \delta$ and thus

$$
\left|\ell\left(\psi_{ \pm}\right)-\ell(h)\right| \leq|\ell(h)| \frac{4 \delta}{1+4 \delta}+\frac{2 \delta}{1+4 \delta} \leq 6 \delta
$$

Consequently,

$$
\operatorname{re} \ell\left(\psi_{ \pm}\right) \geq \operatorname{re} \ell(f)-10 \delta \geq 1-11 \delta \geq 1-\varepsilon
$$

so that $\psi_{ \pm} \in S$; but $\left\|\psi_{+}-\psi_{-}\right\|=2 /(1+4 \delta) \rightarrow 2$ as $\delta \rightarrow 0$. Hence $\operatorname{diam} S=2$.

The point of working with $g$ rather than $g_{1}$ in the proof is to control $\|1-g\|$. Another way to achieve this is to construct a suitable conformal map $\phi$ from the unit disk to a neighbourhood of $[0,1]$ in $\mathbb{C}$ and to consider $\phi \circ g_{1}$.

We now extend Theorem 1 to relatively weakly open subsets.

Theorem 2. Every nonvoid relatively weakly open subset $W$ of the unit ball of an infinite-dimensional uniform algebra $\mathcal{A}$ has diameter 2.

Proof. Every nonvoid relatively weakly open subset of the unit ball of a Banach space contains a convex combination of slices, see [2, Lemma II.1] or [6]. Thus, if $W \subset B_{\mathcal{A}}$ is given as above, there are slices $S^{(1)}, \ldots, S^{(n)}$ and $0 \leq \lambda_{j} \leq 1, \sum_{j=1}^{n} \lambda_{j}=1$, such that $\sum_{j=1}^{n} \lambda_{j} S^{(j)} \subset W$. 
Let $S^{(j)}=\left\{a \in B_{\mathcal{A}}: \operatorname{re} \ell_{j}(a) \geq 1-\varepsilon_{j}\right\}$ with $\left\|\ell_{j}\right\|=1$ and representing measures $\mu_{j}$. We now perform the construction of the proof of Theorem 1 with $\varepsilon=\min \varepsilon_{j}, 0<\delta \leq \varepsilon / 11$ as before and a nonvoid open set $V_{0} \subset K$ such that $\left|\mu_{j}\right|\left(V_{0}\right) \leq \delta$ for all $j$. We obtain functions $h^{(j)}$ and $\varphi$ (independently of $j)$ such that $\left(h^{(j)} \pm \varphi\right) /(1+4 \delta) \in S^{(j)}$ and $\|\varphi\|=1$. Therefore $\sum_{j=1}^{n} \lambda_{j} h^{(j)} \pm \varphi \in(1+4 \delta) W$, and $\operatorname{diam} W=2$.

In case $K$ does not have isolated points, Theorem 1 is a formal consequence of the Daugavet property of $\mathcal{A}$, proved in [8] or [7], and [3, Lemma 2.1]. Likewise Theorem 2 follows from [6].

Corollary 3. The unit ball of an infinite-dimensional uniform algebra does not contain any denting points or merely points of continuity for the identity mapping with respect to the weak and the norm topology.

We would also like to remark that T.S.S.R.K. Rao [5] has shown that $B_{W C(K, X)}$, the unit ball in the space of continuous functions from a compact Hausdorff space into a Banach space equipped with its weak topology, has no denting points. He has also given a proof of Corollary 3 based on techniques from that paper.

\section{REFERENCES}

[1] P. Beneker, J. Wiegerinck. Strongly exposed points in uniform algebras. Proc. Amer. Math. Soc. 127 (No 5) (1999), 1567-1570.

[2] N. Ghoussoub, G. Godefroy, B. Maurey, and W. Schachermayer. Some topological and geometrical structures in Banach spaces. Mem. Amer. Math. Soc. 378 (1987).

[3] V. Kadets, R. Shvidkoy, G. Sirotkin, And D. Werner. Banach spaces with the Daugavet property. Trans. Amer. Math. Soc. (to appear).

[4] G. M. Leibowitz. Lectures on Complex Function Algebras. Scott, Foresman and Company 1970.

[5] T.S.S.R.K. RAO. There are no denting points in the unit ball of $W C(K, X)$. Proc. Amer. Math. Soc. 127 (No 10) (1999), 2969-2973.

[6] R. Shvidkoy. Geometric aspects of the Daugavet property. J. Funct. Anal. (to appear).

[7] D. Werner. The Daugavet equation for operators on function spaces. J. Funct. Anal. 143 (1997), 117-128.

[8] P. Wojtaszczyk. Some remarks on the Daugavet equation. Proc. Amer. Math. Soc. 115 (1992), 1047-1052.

Department of Mathematics, Agder College, Tordenskjoldsgate 65, 4604 KRISTIANSAND, NORWAY

E-mail address: Olav.Nygaard@hia.no

Department of Mathematics, Freie Universität Berlin, Arnimallee 2-6, D-14 195 Berlin, Germany

E-mail address: werner@math.fu-berlin.de 